DOI: $10.19195 / 0137-1169.37 .6$

\title{
La alternancia de los modos subjuntivo e indicativo después de el hecho de que en los periódicos en línea
}

\section{Introducción}

En la mayoría de las gramáticas españolas, tanto modernas como antiguas, encontramos las definiciones del modo subjuntivo basadas en la expresión de la irrealidad, emoción, duda, o bien no aserción y falta de compromiso con la factualidad del enunciado, como, por ejemplo, según Gili Gaya (1973:132): "Usamos el subjuntivo en la oración subordinada siempre que el verbo exprese una acción dudosa, posible, necesaria o deseada".

Las primeras observaciones del subjuntivo eran, sobre todo, de carácter sintáctico y subrayaban la dependencia de este modo. Así lo trató Salvá (1847:167) explicando que "el indicativo expresa la idea del verbo sin dependencia del otro mientras que el subjuntivo tiene que ir unido a otro verbo, bien explícito, bien sobreentendido, que lo determina y con el cual se enlaza por medio de una partícula conjuntiva". Sin embargo, la lingüística moderna suele distinguir entre el modo, entendido como conjugación verbal y la modalidad, es decir un concepto semántico, que denota la actidud del hablante (RAE 2010:474). Han sido numerosos los planteamientos del carácter semántico que trataban de describir la naturaleza del subjuntivo frente al indicativo, en mayoría de casos basándose en la dicotomía del tipo: Certeza/Incertidumbre, Realidad/Irrealidad (RAE 2010:474, Ahern 2008:12, Alarcos Llorach 1994:153), Objetividad/Subjetividad (Hernández Alonso 1986:288) o Aserción/No aserción (RAE 2010: 474, Nowikow 2001:18, Terrel y Hooper 1974).

No obstante, hay que destacar que existen también contextos en los que se emplea el subjuntivo en las cláuslas que no expresan ni emoción ni falta de aserción, 
pues las dicotomías mencionadas arriba no son suficientes para poder explicarlas. Nos referimos aquí a la selección modal después de la expresión el hecho de que, cuyo carácter, como subraya Lipski (1978:931), es factual y no está relacionado con la expresión de realidad e irrealidad o duda. A pesar de que la alternancia de subjuntivo e indicativo en las oraciones sustantivas ha sido ampliamente indagada, el empleo de los modos con la expresión el hecho de que sigue siendo un objeto que merece un estudio más profundo, arraigado en un contexto amplio y contemporáneo.

Por lo tanto, el objetivo del presente artículo es observar la alternancia del subjuntivo e indicativo después de la expresión el hecho de que basándose en los ejemplos de la prensa contemporánea (el Mundo, el País, BBC), tratando de destacar los factores que determinan la selección modal, teniendo en cuenta el nivel textual- informativo de los enunciados.

\section{El modo desde el punto de vista de la teoría de relevancia}

Como hemos mencionado anteriormente, el planteamiento de Terrel y Hooper (1974) fue pioniero en tratar los modos desde el punto de vista semántico. Dichos lingüístas establecieron la distinción entre la presuposición y la afirmación, o bien la aserción, a partir de lo que describieron las relaciones entre noción semántica, clase verbal y selección de modo. De ahí que asociaran el empleo del indicativo con la aserción (tanto aserción fuerte como débil) y el empleo del subjuntivo con la presuposición. No obstante, este estudio despertó mucha polémica, por ejemplo de Manteca Alonso-Cortés (1981), Mejías Bikandi (1994) o Rivero (1971), según los cuales la propuesta de Terrel y Hooper puede resultar ineficaz, ya que no explica todos los contextos de la selección modal. Aun así, fue el planteamiento que dio paso a otras teorías semántico- pragmáticas, que abarcaron el nivel informativo del enunciado, las que pueden arrojar la luz sobre el problema bajo el presente estudio.

Ante todo, se debe reconocer que "las formas del indicativo y subjuntivo se utilizan como transmisores y repartidores de información" (Nowikow 2001:71). Por consiguiente, se tiene que prestar especial atención a las funciones pragmáticas de los modos en español. Partiendo de la teoría de relevancia presentada por Sperber y Wilson en 1986, podemos discernir entre la información dada o compartida y la información nueva o ignorada, asumiendo el criterio de la novedad (íbid.). Además, desde el punto de vista del criterio de la relevancia/importancia, distinguimos entre la información principal o relevante y la información secundaria o irrelevante. Los numerosos análisis de la selección modal llevados a cabo desde el punto de vista pragmático, conducen a la conclusión de que el indicativo suele emplearse como portador de información nueva/relevante, mientras que el subjuntivo marca la información vieja/compartida/irrelevante (íbid. 71-72). 
Al tratar el modo desde la perspectiva del discurso, debemos recordar la distinción establecida por Mathesius a mediados del siglo XX entre el tema, es decir el objeto del discurso y el rema, la información aportada al tema (Nowikow 2001:72). Posteriormente surgieron también otras nociones semejantes, como tópico o soporte y aporte, pero todos se pueden identificar con la dicotomía entre información relevante e irrelevante (íbid.). Como veremos más adelante, estos conceptos resultan de suma importancia para delimitar la alternancia del subjuntivo e indicativo después de la expresión el hecho de que.

Es digna de atención la propuesta de Lunn (1989), la que presentó en el artículo titulado "Spanish Mood and Prototype of Assertability". En dicho artículo, la autora no descarta la validez de la hipótesis de Terrel y Hooper de que el subjuntivo es el modo de no aserción, aunque reprocha que el estudio no explica el porqué de este problema. Propone el término de prototype of assertability, señalando que el subjuntivo en español se usa no solo para la información que no es verdadera o no realizada (como en los ejemplos 1-3), sino también para expresar lo verdadero y lo real (como en los ejemplos 4-5) (Lunn 1989:688). Véase:

1) No es verdad que venga.

2) Hablaremos cuando vuelvas.

3) Quiero que vengas.

4) El hecho de que vengas es importante.

5) Estoy contento de que vengas.

La autora (1989:690) analiza el uso del subjuntivo a la luz de la teoría de relevancia de Sperber y Wilson y llega a la conclusión que el modo subjuntivo muestra la información como poco relevante o irrelevante, lo que se debe al hecho de que no aportan nada nuevo para mejorar la representación del mundo del oyente. Esta característica se refiere tanto a la información no verdadera como a la información presupuesta. Además, Lunn sostiene que la subjunctivizable information se debe carecterizar por los rasgos que no posee (ibíd., 691), dado que la información aseverada se entiende como la información que es fiable, desde el punto de vista del valor veritativo, e informativa, desde el punto de vista de la novedad. La alternancia del subjuntivo e indicativo se presenta de la manera siguiente:

Tabla 1: Prototype of Assertability según Lunn (1989:691)

\begin{tabular}{|ccc|}
\hline $\begin{array}{c}\text { Less assertable } \\
\text { Untrue }\end{array}$ & Assertable & Less assertable \\
Both true and new & Old \\
\hline SUBJUNCTIVE & INDICATIVE & SUBJUNCTIVE \\
\hline
\end{tabular}

Para el presente artículo, es interesante la observación de Haverkate (2002:88-90) que atribuye al modo la función discursiva de organizar la información, situando 
la información más relevante en el primer plano, y la información poco relevante en el segundo plano. Es de esperar que esta característica del modo se pueda notar, sobre todo, en el registro periodístico, puesto que, como describe Lunn (1989:693), los modos en la prensa tienden a encauzar la atención del lector. Además, observó que en títulos de las noticias se tiende a utilizar el indicativo, mientras que al repetir la misma información dentro del texto, se suele emplear el subjuntivo (íbid.).

Otra lingüísta que llegó a conclusiones afines fue Levandera (1983), que al investigar el uso del subjuntivo en el habla grabada en las calles de Buenos Aires, observó que su empleo puede desempeñar la función anafórica, "desfocalizando" la información ya conocida por el hablante, como se puede ver en el fragmento siguiente (1983:337):

Entonces yo le dije "Mira, Gerardo, ¿a vos te falta algo? Me dijo "No me falta nada". Digo "Mientras que a vos no te falte nada, como vos decís [...]

Por consiguiente, podemos concluir en palabras de Jary (2002:170): “[...] the central difference between the indicative and subjunctive moods in Spanish is that while the former may be used to present the proposition it expresses as relevant in its own right, the latter cannot; [...] this explains the crucial differences in how the two moods are interpreted".

\section{Las observaciones acerca de la alternancia modal después de la expresión el hecho de que}

Hay que destacar que el uso del subjuntivo después de la expresión el hecho de que no sirve ni para expresar duda ni emoción, sino tiene carácter factual, ya que la expresión no es sinonímica a la posibilidad de (Lipsky 1978:931). Veamos un ejemplo:

6) [...] Catalá ha descartado que el pasado político de Andrés Ollero — que fue diputado del PP durante 17 años-le inhabilite para presidir la institución. En opinión del ministro el hecho de que haya sido diputado no supone una ,tacha” $o$,,desdoro" sino que demuestra un compromiso con las instituciones que hay que „reconocer”. El País, 21.02.2017 (1).

En el ejemplo presentado arriba, se emplea el subjuntivo, a pesar de que no cabe duda de que la información es verdadera y real, ya que esto se manifiesta en la frase precedente (fue diputado). Además, atendiendo al contexto, la información utilizada con el hecho de que, no es una información nueva. Por el contrario, es una 
información repetida, ya conocida por el lector y se la menciona de nuevo solo con el objetivo de comentarla. Así que no se puede desdeñar aquí la función discursiva del modo, entendida desde el punto de vista de la teoría de la relevancia, como lo describió Lunn (1989) o Jary (2002). Por otro lado, podríamos asociarlo con la dicotomía tema/rema o soporte/aporte, donde la información que el político ha sido diputado constituiría el soporte, mientras que la información expresada en la segunda parte de la frase constituiría el aporte, es decir la información nueva, relevante.

En el Manual de la Nueva gramática de la lengua española de la RAE (2010:481) también se recalca carácter especial de la expresión el hecho de que: "En el caso de los sustantivos abstractos hecho [...] (en las estructuras del tipo el hecho de que [...]), la alternancia modal puede ponerse en relación con la influencia del verbo principal, pero también con la estructura informativa de la oración, dado que el indicativo aporta la información nueva o relevante en un fragmento del discurso [...]. El subjuntivo se asocia, en cambio, con la información temática (en el sentido de presentada como conocida) [...]."

Curiosamente, la alternancia modal con el marcador el hecho de que no ha suscitado el interés de los investigadores, como otras expresiones con las cuales se alternan subjuntivo e indicativo, como las expresiones de duda o emoción. Indudablemente, cabe destacar el artículo titulado "Selección del modo después de el hecho de que" de Krakuskin y Cedeño (1992) cuyo análisis fue basado en los artículos de Mariano Grondona, periodista de "La Revista Visión" (México) o el artículo de Lipsky (1978) "Subjunctive as Fact?". Ambos artículos enfatizan la necesidad de llevar a cabo un análisis en un contexto más amplio.

La dificultad que aparece en el análisis del modo después de la expresión el hecho de que es la posibilidad de usar no solo el subjuntivo sino también el indicativo. Sin embargo, como nota Lipski (1978:932), los hablantes nativos no son muy decididos a la hora de elegir el modo en este caso. Según Fente, Fernández y Feijoó (1972:54) "en la mayoría de los casos, esta expresión admite indistintamente los dos modos, por lo que puede considerarse un caso más de neutralización, aunque existe una tendencia decidida en el habla cuidada a usar el subjuntivo". No obstante, Borrego et al. (1985:107) subrayan que la afirmación de que esta expresión rige el subjuntivo "provoca la perplejidad de quienes defienden que los hechos reales [...] son el campo ideal de actuación del indicativo". A nuestro juicio, la explicación que proporcionan a continuación podría servir mejor para razonar el uso del subjuntivo en el ejemplo (6), ya que los autores sostienen que el uso del subjuntivo en este caso se debe al hecho de que normalmente es presentador de algo ya conocido. No obstante, Borrego et al. (íbid.) añaden que el empleo del subjuntivo con el hecho de que puede también desempeñar el papel de presentador de algo cuya realización se ve eventual o hipotética. Para ilustrarlo, se presenta la frase siguiente (íbid.): 
7) "Márchate si quieres. El hecho de que te marches no me va a hacer cambiar de opinión".

Tanto Borrego et. al (1985:108) como Krakuskin y Cedeño (1992:1290) aluden a la importancia de la posición de la expresión el hecho de que en la oración, manifestando que si este marcador se encuentra en la posición inicial de la oración es seguido por el subjuntivo. Esto se debe a que, según presenta Contreras (1976:26) en su investigación sobre el orden de palabras en español, el rema del rango más alto se suele colocar en la posición final de la oración. De hecho, hemos notado esta tendencia en los textos de prensa analizados. Cuando el marcador el hecho de que aparece en la posición inicial, se usa el subjuntivo, como en los ejemplos (10), (12), (13), mientras que cuando se pospone al verbo principal, se emplea el indicativo, como en los ejemplos (15), (16), (17), (18), (19). Sin embargo, observamos también excepciones (ejemplos (11), (21)), las que trataremos de justificar más adelante.

Así pues, Krakuskin y Cedeño (1992:1290-1291) concluyen que Grondona usa el indicativo para marcar la información nueva de alto valor informativo. Por oposición, el subjuntivo se ve ligado al poco valor informativo. No obstante, Lipski (op cit.) señala que la convicción de que el indicativo después de el hecho de que se usa solo para indicar la información nueva, no siempre es verdadera. Lo corrobora en los ejemplos siguientes (op cit. 932):

8) "El presidente no se fijó en el hecho de que el hambre todavía existe en este pais."

9) “Cómo explicas el hecho de que nadie te quiere?”

El autor explica que el enunciado (8) podría decirse en una conferencia sobre el hambre, en la que el presidente pasó por alto un hecho ya conocido y comentado por otros oradores. El (9) podría ser dicho por un amigo que quiere consolar al otro, poniendo en tela de juicio la naturaleza de la situación (íbid.).

\section{Análisis de ejemplos}

Para llevar a cabo el análisis de la selección modal después de el hecho de que hemos elegido los textos de prensa virtual, basándonos en artículos de el País (www. elpais.com), el Mundo (www.elmundo.es), BBC (www.bbc.com/mundo) en sus versiones en línea. Con el objetivo de encontrar ejemplos del uso del subjuntivo/ indicativo después de el hecho de que, hemos analizado aproximadamente cien artículos en los que aparecía dicho marcador. Los artículos provenían de los periódicos mencionados, pero sobre todo de el País, del periodo del tiempo entre enero y junio del 2017. Posteriormente hemos escogido unos ejemplos, tanto con el uso 
del subjuntivo como del indicativo (véase la lista de artículos), para resumir nuestras conclusiones acerca del tema estudiado. Cabe subrayar que nuestra intención es justificar la selección modal después de el hecho de que, sin fijarnos en la frecuencia de la aparición de un modo u otro, por lo tanto los ejemplos seleccionados nos parecen suficientes para reflejar la naturaleza del fenómeno. Hemos elegido este tipo de material, tomando en consideración varios factores. Sobre todo, este tipo del análisis nos permite ver la selección modal desde el punto de vista discursivo-informativo, lo que parece crucial, dada la naturaleza del objeto de estudio. Además, la lengua que se presenta en estos textos es el castellano contemporáneo, vivo, con lo cual esperamos sacar conclusiones sobre el actual uso del subjuntivo/ indicativo con el hecho de que.

Al principio, observemos las situaciones en las que los autores de artículos emplearon el subjuntivo. Cabe recordar que muchos gramáticos, como mencionados anteriormente Fente, Fernández y Feijoó (op cit.), sostienen que el uso del subjuntivo es el que prevalece después del marcador el hecho de que.

En muchos de los casos analizados, notamos que el subjuntivo tiende a marcar la información ya conocida, menos relevante, antes mencionada en el texto, o bien en el titular, o bien a lo largo del artículo. Se puede notar que este uso tiene la función anáforica, ya que se vuelve a exponer la información precedentemente enfatizada. La razón de repetirla en el discurso reside en el hecho de que se procura comentarla, criticarla o desarrollarla. Así pues, se confirma que la selección del modo se presenta como una estrategia discursiva cuyo objetivo es focalizar y "desfocalizar" la atención del lector. Si nos fijamos en el orden de palabras, podemos constatar que el subjuntivo suele colocarse al inicio del enunciado, presentando el tema / el soporte, como en los ejemplos (10) y (12). Sin embargo, no tiene que ser necesariamente así, como veremos en el (11). Obsérvese:

10) [...] La votación de esta enmienda ha provocado una intensa polémica en el PP y una creciente impresión de pucherazo porque se rechazó „, a ojo” $y$, posteriormente, se ofreció un recuento. [...] El hecho de que la mesa de la Ponencia Política y de Estatutos rechazara la enmienda ,a ojo” pese a la casi igualdad de sies y de noes en la votación, provocó ayer protestas de los compromisarios. El mundo, 11.02.2017 (2).

11) [...] con la recuperación de la economía, otros sectores como el inmobiliario, el de coches o el de electrodomésticos, están vendiendo más y mejor, con el de alimentos y bebidas está pasando lo contrario [...]. Hay otro factor que influye en el hecho de que los supermercados havan vendido menos y es que los españoles, con la crisis, nos hemos vuelto más responsables y tiramos menos comida que antes. [...] Hay un factor demográfico que también ha influido en el hecho de que los supermercados e hipermercados vendieran menos en 2016 y es el envejecimiento de la población. El mundo, 6.02.2017 (3). 
12) La ONU tuvo que proteger a la ONG Global Witness para que sus representantes no fueran detenidos por revelar vínculos del partido oficial con proyectos hidroélectircos que han causado decenas de muertes. [...], El $\underline{\text { L }}$ hecho de que la ONU me tuviera que rescatar quiere decir que el gobierno tiene miedo de los hallazgos [...], defiende el representante de Global Witness. El País, 11.02.2017 (4).

Encontramos también usos del subjuntivo después del marcador el hecho de que, aunque la información no ha aparecido anteriormente en el texto. En tales casos, el autor del texto supone que es una información conocida por el lector, ya que son informaciones del dominio público, del conocimiento general, a los que se alude, sobre todo, para comentarlas. Son de poca relevancia, puesto que no mejoran la representación del mundo del lector. Por ejemplo, el ejemplo (13) corresponde a un juicio generalmente conocido, se supone que todos los lectores poseen este conocimiento, por lo cual, se tacha la información de menos relevante. El ejemplo (14) es un fragmento del artículo que analiza la gala de los galardones Goya, y aunque el autor no menciona en ninguna parte anterior del texto el hecho subrayado, considera que los lectores, como han visto la gala, están enterados del tema principal y por lo tanto, poseen el conocimiento del hecho. Observemos:

13) El hecho de que el impacto de las actividades humanas sobre el medioambiente sea ampliamente conocido hoy en dia se debe, en parte, al trabajo de los ecólogos Gene Likens [...] y Marten Scheffer [...]. El País, 7.02.2017 (5).

14) [...] fueron demasiados los encuadres de cámara extraños, que iban $y$ venian o que no enfocaban debidamente. $O$, $[\ldots]$ el hecho de que en televisión estuviéramos viendo mucho mejor al violonchelista que a los propios recordados, que iban desfilando de fondo de manera que solo se podía ver de forma correcta el nombre y rostro de algunos de ellos. El País, 6.02.2017 (6).

Respecto al uso del indicativo después de el hecho de que, podemos observar que el planteamiento de Krakuskin y Cedeño (1992:1291), según los cuales "el indicativo se da en las clásulas factivas cuando éstas contienen proposiciones de alto valor informativo", tiene reflejo también en los textos de prensa analizados en este artículo. Se desprende de aquí que el uso del indicativo sirve para marcar que la información será de suma importancia en el discurso, y que probablemente no es todavía conocida por el lector. Veamos un ejemplo:

15) Es claro que la declaración del exsenador, y la mención también del nombre de Roberto Prieto por parte de otro de los involucrados en la recepción de dineros de Odebrecht como el viceministro Gabriel Garcia 
no son suficiente prueba por si solas pero si lo es el hecho de que la plata en cuestión fue monetizada en Colombia. El País, 11.02.17 (7).

El ejemplo (15) presentado arriba proviene de un artículo que aborda el tema de la corrupción en Colombia y el hecho subrayado trae una información nueva, no mencionada previamente, arrojando la nueva luz sobre el asunto descrito. Por lo tanto, el uso del indicativo aquí focaliza la atención del lector, enfatiza la relevancia del mensaje. El uso del indicativo con un objetivo semejante lo podemos apreciar también en los ejemplos siguientes:

16) [...] no hay una relación bilateral más importante que la de Pekín con Washington y el hecho de que desde que asumió la presidencia en enero Trump no había conversado con su homólogo chino, había provocado un creciente escrutinio. $\mathrm{BBC}, 11.02 .17$ (8).

17) "El peor grupo del mundo" viene del hecho de que yo siempre me he considerado un periodista musical frustrado porque me encanta ese tipo de periodismo [...]. El Mundo, 11.02.17 (9).

18) ¿Cuál es el sentido para PSA de quedarse con semejante perla? La respuesta se encuentra en el hecho de que la francesa no aspira a ser una gran automovilística mundial. Quiere ganar Europa. El País, 26.02.17 (10).

19) La fiscalía también habia argumentado que el riesgo de fuga quedaba mitigado por el hecho de que el esposo de la Infanta cuenta con escolta policial. El País, 24.02.17 (11).

No obstante, hemos también observado unos ejemplos del uso del indicativo con el hecho de que que parecen contradecir lo demostrado por Krakuskin y Cedeño (op cit.). El ejemplo (20) procede de un artículo cuyo tema principal es la presunta incomodidad que siente Ricky Rubio, jugador de baloncesto, hacia su nuevo entrenador, Thibodeau. El asunto ya se menciona en la cabecera del artículo, después varias veces dentro del texto. Podemos notar que el texto gira en torno de esta noticia, a pesar de lo cual en la frase con el hecho de que se utiliza el modo indicativo:

20) Título: El base logra sus mejores números pese a los rumores sobre su traspaso y su incomodidad con Thibodeau. [...] Un periodista del Minnesota Post le preguntó a Thiboudeau: "Se ha hablado mucho sobre el hecho de que usted y Ricky no se han sentido cómodos el uno con el otro desde el principio. ¿Hay algo de cierto en eso?". A lo que el entrenador de los Wolves contestó: "No. Mi trabajo es exponer el plan y decirle la verdad tal como la veo cada día. El País, 10.02.17 (12). 
Se supone que el uso del indicativo en este caso tiene que ver con la polémica que se suscita acerca del hecho referido. La información podría ser relevante, pero el contexto nos induce a pensar que es solo un rumor e incluso el mismo interesado tampoco lo confirma. El uso presentado aquí parece semejante al uso del indicativo en el ejemplo (9) propuesto por Lipski (op cit. 933), el cual también justifica el uso del indicativo diciendo que el hablante, analizando la situación, pone el hecho en tela de juicio. Por consiguiente, es posible el uso del indicativo con el hecho de que para mencionar los hechos ya conocidos y previamente comentados (íbid.).

Siguiendo a Contreras (op cit.), como ya hemos señalado, el rema se suele manifestar en la segunda parte de la oración, posponiéndose al tema. Entonces, deberíamos esperar el uso del subjuntivo si la expresión el hecho de que aparece en la posición inicial en la oración, pero no es siempre así:

21) El hecho de que en esta era ganar Eurovisión se ha demostrado casi inviable para España año tras año, abre también de forma recurrente el debate sobre si es conveniente y rentable seguir participando. El Mundo, 10.02.17 (13).

La información transmitida en el ejemplo (21) se menciona en el artículo por primera vez, aunque no parece una información totalmente nueva para los lectores, especialmente los interesados en el tema. A nuestro juicio, el uso del indicativo aquí, a pesar de la posición inicial en la frase, sirve para poner de relieve el problema del fracaso de los cantantes españoles en el concurso. Más aún, la frase citada abre un párrafo nuevo, en el que se comenta el objetivo de participar en Eurovisión, de ahí que la información expresada en indicativo sea aquí muy relevante.

\section{Conclusiones}

En el caso de la alternancia de los modos subjuntivo e indicativo después de la expresión el hecho de que, no se puede soslayar su función discursivo-informativa, vista desde el punto de vista de la teoría de relevancia de Sperber y Wilson (1986). Hemos probado que la selección del modo en las clásulas factivas está estrechamente relacionada con el valor informativo de la oración, tal como lo describieron Krakuskin y Cedeño (op cit.). Por lo tanto, en el análisis es imprescindible tomar en consideración todo el contexto, puesto que los modos sirven para focalizar y "desfocalizar" la atención del hablante (Haverkate, op. cit.). En las oraciones analizadas, el indicativo tiende a marcar mayor valor informativo del enunciado, por lo que suele ser utilizado en la segunda parte de la oración, denotando el rema. Por oposición, el subjuntivo parece disminuir la relevancia de la información y en la mayoría de casos manifiesta la información ya conocida, que no trae nada nuevo a la representación del mundo del receptor. Entonces, se puede encontrar 
el uso del subjuntivo con el hecho de que en la posición inicial de la oración. Sin embargo, a partir del corpus estudiado hemos observado, acorde con lo que puntualizó Lipski (op cit.), que se pueden notar también los usos del indicativo con la información conocida y anteriormente mencionada. Más aún, la selección del modo en este caso parece una decisión bastante individual del hablante/autor que se ve influida por razones pragmáticas. Es de subrayar el carácter peculiar del uso del subjuntivo en este tipo de oraciones en la lengua española, ya que es de índole diferente al uso del subjuntivo más común, es decir, de duda, sentimiento o comentario, dado que aquí el subjuntivo se emplea para expresar la realidad.

\section{Referencias bibliográficas}

AHERn Aoife, 2008, El subjuntivo: contextos y efectos, Madrid.

ALARCos LloraCH Emilio, 1994, Gramática de la lengua española, Madrid.

Borrego Nieto Julio / Gómez Asencio José Jesús / Prieto DE LOS Mozos Emilio, 1985, El subjuntivo. Valores y usos, Madrid.

CONTRERAs Heles, 1976, A Theory of Word Order with Special Reference to Spanish, Amsterdam.

Fente Gómez Rafael / Alvarez Fernández Jesús / FeIJóo Lope, 1972, El subjuntivo, Madrid.

GILI Gaya Samuel, 2002, Curso superior de sintaxis española, Barcelona.

HaverKate Henk, 2002, The Syntax, Semantics and Pragmatics of Spanish Subjunctive, Amsterdam/Philadelphia.

JARY Mark, 2002, Mood in relevance theory: a re-analysis focusing on the Spanish subjunctive, en: UCL Working Papers in Linguistics 14, pp. 157-187.

KRAKUSKIN Margarita / CEDEÑo Aristófanes, 1992, Selección del modo después de el hecho de que, en: Hispania 75, n. 5, pp. 1289-1293.

LEVANDERA Beatriz, 1983, Shifting mood in Spanish discourse, en: Klein-Andreu F. (ed.), Discourse Perspectives on Syntax, New York, pp. 209-236.

LIPSKI John M., 1978, Subjunctive as Fact?, en: Hispania 61, n. 4, pp. 931-934.

LunN Patricia V., 1989, Spanish mood and the prototype of assertability, en: Linguistics 27, pp. 687-702.

MANTECA Alonso-Cortés Ángel, 1981, Gramática del subjuntivo, Madrid.

MEJíAS BIKANDI Errapel, 1994, Assertion and Speaker's Intention: A Pragmatically Based Account of Mood in Spanish, en: Hispania, 77 n. 4, pp. 892-902.

NowIKOW Wiaczesław, 2001, La alterancia de los modos Indicativo y Subjuntivo en las clásulas subordinadas sustantivas, Poznań.

Real Academia Española y Asociación de Academias de la Lengua Española, 2010, Nueva gramática de la lengua española. Manual, Madrid.

RIVERo María Luisa, 1971, Mood and Presupposition in Spanish, en: Foundations of Language 7, pp. 305-336.

SALVÁ Vicente, 1847, Gramática de la lengua castellana según se habla ahora, Valencia.

SPERBER Dan / WiLSON Deirdre, 1986, Relevance: Communication and Cognition, Cambridge.

TERRELL Tracy / HoOPER Joan, 1974, A semantically based analysis of mood in Spanish, en: Hispania 57, n. 3 , pp. $484-494$. 


\section{Lista de artículos de los que proceden ejemplos analizados (Fecha de consulta: 30.06.2017)}

(1) http://politica.elpais.com/politica/2017/02/21/actualidad/1487671264_739300.html.

(2) http://www.elmundo.es/espana/2017/02/11/589ee02d268e3ef11 f8b4830.html.

(3) http://www.elmundo.es/economia/2017/02/06/5898654fe5fdea71328b4629.html.

(4) http://internacional.elpais.com/internacional/2017/02/09/america/1486617921_790115.html.

(5) http://elpais.com/elpais/2017/02/07/ciencia/1486468000_302447.html.

(6) http://cultura.elpais.com/cultura/2017/02/05/television/1486252862_288118.html.

(7) http://internacional.elpais.com/internacional/2017/02/11/colombia/1486830228_685624.html.

(8) http://www.bbc.com/mundo/noticias-internacional-38929520.

(9) http://www.elmundo.es/comunidad-valenciana/alicante/2017/02/11/589de85546163f92328b4586. $\mathrm{html}$.

(10) http://economia.elpais.com/economia/2017/02/24/actualidad/1487939700_285115.html.

(11) http://politica.elpais.com/politica/2017/02/23/actualidad/1487837888_735616.html.

(12) http://deportes.elpais.com/deportes/2017/02/10/actualidad/1486756273_911104.html.

(13) http://www.elmundo.es/television/2017/02/10/589e161622601dd20a8b46aa.html. 